Research article

\title{
Risk factors for breast cancer characterized by the estrogen receptor alpha A908G (K303R) mutation
}

\author{
Kathleen Conway ${ }^{1,2}$, Eloise Parrish², Sharon N Edmiston², Dawn Tolbert², Chiu-Kit Tse ${ }^{1}$, \\ Patricia Moorman ${ }^{3}$, Beth Newman ${ }^{4}$ and Robert C Millikan ${ }^{1,2}$
}

\begin{abstract}
1Department of Epidemiology, School of Public Health, CB 7435, University of North Carolina, Chapel Hill, NC 27599, USA
2 Lineberger Comprehensive Cancer Center, School of Medicine, CB 7295, University of North Carolina, Chapel Hill, NC 27599, USA

${ }^{3}$ Department of Community and Family and Preventive Medicine, Duke University School of Medicine, Box 2949, Durham, NC 27710, USA

${ }^{4}$ School of Public Health, Institute for Health and Biomedical Innovation, Queensland University of Technology, Brisbane 4059, Australia
\end{abstract}

Corresponding author: Kathleen Conway, kconway@med.unc.edu

Received: 5 Jan 2007 Revisions requested: 21 Feb 2007 Revisions received: 25 May 2007 Accepted: 6 Jun 2007 Published: 6 Jun 2007

Breast Cancer Research 2007, 9:R36 (doi:10.1186/bcr1731)

This article is online at: http://breast-cancer-research.com/content/9/3/R36

(C) 2007 Conway et al.; licensee BioMed Central Ltd.

This is an open access article distributed under the terms of the Creative Commons Attribution License (http://creativecommons.org/licenses/by/2.0), which permits unrestricted use, distribution, and reproduction in any medium, provided the original work is properly cited.

\begin{abstract}
Introduction Estrogen is important in the development of breast cancer, and its biological effects are mediated primarily through the two estrogen receptors alpha and beta. A point mutation in the estrogen receptor alpha gene, ESR1, referred to as A908G or K303R, was originally identified in breast hyperplasias and was reported to be hypersensitive to estrogen. We recently detected this mutation at a low frequency of $6 \%$ in invasive breast tumors of the Carolina Breast Cancer Study (CBCS).
\end{abstract}

Methods In this report, we evaluated risk factors for invasive breast cancer classified according to the presence or absence of the ESR1 A908G mutation in the CBCS, a population-based case-control study of breast cancer among younger and older white and African-American women in North Carolina. Of the 653 breast tumors evaluated, 37 were ESR1 A908G mutationpositive and 616 were mutation-negative.

Results ESR1 A908G mutation-positive breast cancer was significantly associated with a first-degree family history of breast cancer (odds ratio $[O R]=2.69,95 \%$ confidence interval $[\mathrm{Cl}]=1.15$ to 6.28 ), whereas mutation-negative breast cancer was not. Comparison of the two case subgroups supported this finding ( $O R=2.65,95 \% \mathrm{Cl}=1.15$ to 6.09 ). There was also the suggestion that longer duration of oral contraceptive (OC) use $\left(\mathrm{OR}=3.73,95 \% \mathrm{Cl}=1.16\right.$ to $12.03 ; P_{\text {trend }}=0.02$ for use of more than 10 years) and recent use of OCs (OR $=3.63,95 \%$ $\mathrm{Cl}=0.80$ to $16.45 ; P_{\text {trend }}=0.10$ for use within 10 years) were associated with ESR1 A908G mutation-positive breast cancer; however, ORs for comparison of the two case subgroups were not statistically significant. Hormone replacement therapy use was inversely correlated with mutation-negative breast cancer, but the effect on mutation-positive cancer was unclear due to the small number of postmenopausal cases whose tumors carried the mutation. Mutation-negative breast cancer was associated with several reproductive factors, including younger age at menarche (OR $=1.46,95 \% \mathrm{Cl}=1.09$ to 1.94$)$ and greater total estimated years of ovarian function $(\mathrm{OR}=1.82$, $95 \% \mathrm{Cl}=1.21$ to 2.74$)$.

Conclusion These preliminary results suggest that OCs may interact with the ESR1 A908G mutant receptor to drive the development of some breast tumors.

\section{Introduction}

Most major risk factors for breast cancer are hormonal or reproductive factors that increase exposure to estrogen and/ or progesterone [1]. The importance of estrogen in breast cancer development is also supported by studies demonstrating the occurrence of marked changes in estrogen signaling and in the expression of the two estrogen receptors (ERs), ER alpha and ER beta, during breast tumorigenesis and progression [2-8].

Although mutations in the gene encoding ER alpha, ESR1, are uncommon in primary breast tumors [3], a specific point mutation that occurs at nucleotide 908 within codon 303 and that is referred to as $A 908 \mathrm{G}$ or $\mathrm{K} 303 \mathrm{R}$ was described several

$\overline{\mathrm{AFFTP}}=$ age at first full-term pregnancy; $\mathrm{BMI}=$ body mass index; CBCS = Carolina Breast Cancer Study; $\mathrm{Cl}=$ confidence interval; ER = estrogen receptor; $E S R 1=$ estrogen receptor alpha; $F F P E=$ formalin-fixed paraffin-embedded; $\mathrm{HRT}=$ hormone replacement therapy; $\mathrm{OC}=$ oral contraceptive; $\mathrm{OR}=$ odds ratio. 
years ago by Fuqua and colleagues [9] in one third of typical breast hyperplasias. The A908G mutation affects the border of the hinge and the hormone-binding domains of ESR1 and results in an amino acid change of lysine to arginine (K303R). Compared with the wild-type receptor, the A908G mutant exhibited hypersensitivity to estrogen and was associated with increased cellular proliferation at sub-physiologic levels of estrogen [9]. The A908G mutant receptor displayed similar affinity for estradiol as wild-type receptor but showed enhanced binding to the TIF-2 (transcription intermediary factor-2) coactivator at low hormone levels [9]. More recent studies have also shown that the ESR1 A908G mutation in codon 303 increases phosphorylation at the Ser305 residue through the P13 kinase/Akt signaling cascade [10], protein kinase A [11], and p21-activated kinase [10], but the downstream functional effects of this phosphorylation remain unclear.

We recently detected the ESR1 A908G mutation at a low frequency of $6 \%$ in the primary invasive breast tumors of the Carolina Breast Cancer Study (CBCS), a population-based case-control study of mostly early stage breast cancer in North Carolina [12]. This mutation was confirmed to be somatic in nature and not a germline variant. Mutation-positive tumors were more likely to have mixed lobular/ductal histology and combined grade II (versus grade I) compared with mutationnegative tumors.

The presence of the ESR1 A908G mutation in both breast hyperplasias and invasive carcinomas suggests that it may be an early genetic defect present in the breast tissue of some women and that it, alone or in conjunction with environmental factors, may help to drive the development of some breast tumors. The existence of distinct subtypes of breast cancer as defined by differences in gene expression profiles and in expression of ER and other biomarkers has now been clearly established $[13,14]$. These subtypes vary in their clinical prognosis and may also be characterized by differences in risk factor profiles. Given the reported hypersensitivity of the mutant ESR1 A908G mutant receptor to estrogen, it was of interest to determine whether hormonal risk factors in the CBCS might be associated with breast cancer characterized by the presence or absence of the ESR1 A908G mutation. In particular, we evaluated exposure to exogenous hormones, such as oral contraceptives (OCs) or hormone replacement therapy (HRT), as well as reproductive factors linked to greater endogenous estrogen and progesterone exposure. Our findings suggest that $\mathrm{OC}$ use and first-degree family history of breast cancer may be associated with ESR1 A908G mutation-positive breast cancer, whereas reproductive factors associated with longer exposure to endogenous hormones may be related to mutation-negative breast cancer.

\section{Materials and methods Study population}

This study used subjects and data from phase 1 of the CBCS, a population-based, case-control study of invasive breast cancer conducted among African-American and white women (age range, 20 to 74 years) residing in a 24-county area in central and eastern North Carolina [15]. Both cases and controls were sampled using a modification of randomized recruitment [15]. Sampling probabilities ensured approximately equal samples among cases and controls in the four age-race groups: younger (age range, 20 to 49 years) African-American women, older (age range, 50 to 74 years) African-American women, younger white women, and older white women. Details of recruitment of participants and response rates have been published previously [15]. Incident cases of breast cancer diagnosed between 1 May 1993 and 30 May 1996 were eligible as cases and were identified using the North Carolina Central Cancer Registry's Rapid Case Ascertainment System; of these, 861 patients with breast cancer consented to participate in the CBCS. Clinical data and information on tumor characteristics were obtained from medical records or direct histopathologic review of tumor tissue as described [14]. Controls were drawn from North Carolina Division of Motor Vehicle lists for women ages 20 to 64 years and US Health Care Financing Administration lists for women ages 65 to 74 years and were frequency matched to cases by race and age (in 5year age categories). A total of 790 controls were eligible for and consented to participate in the CBCS. All aspects of this research were approved by the Institutional Review Board of the University of North Carolina School of Medicine.

Tumor tissues and analysis of the ESR1 A908G mutation Formalin-fixed paraffin-embedded (FFPE) tumor blocks were obtained from pathology departments at participating hospitals for 798 of the 861 breast cancer cases. Of these, 684 were of sufficient size to allow for the sectioning of $10-\mu \mathrm{m}$ thick tissue sections for molecular analyses. Tumors were sectioned and underwent standardized histopathologic review as previously described [16]. With the hematoxylin-and-eosinstained slide as a guide, the area of tumor was microdissected away from other surrounding non-tumor tissue, and DNA lysates were prepared using proteinase $\mathrm{K}$ extraction.

Of the 684 tumors available for molecular studies, 653 were successfully screened for mutations in a 104-base pair region of exon 4 surrounding codon 303 of ESR 1 by using a combination of SSCP (single-strand conformational polymorphism) and phosphorus-33-cycle DNA sequencing as described previously [14]. The tumors that were evaluated for the ESR1 A908G mutation were more likely to be later stage $(P=$ $0.005)$, of larger size $(P=0.0002)$, and lymph node-positive $(P=0.006)$ and to exhibit higher combined grade $(P=0.04)$ than tumors that were not screened, consistent with the greater availability of tumor tissue from larger breast tumors. However, the cases screened for mutations did not differ from 
those that were not screened based on age $(P=0.42)$, menopausal status $(P=0.90)$, race $(P=0.63)$, ER status $(P=$ $0.68)$, or tumor histology $(P=0.48)$.

\section{Risk factor information}

Data were obtained from cases and controls during in-person interviews conducted by female registered nurses. The nurseinterviewer elicited information on demographics and potential breast cancer risk factors. Interviews were completed for $77 \%$ ( $n=861$ ) of eligible and locatable cases and for $68 \%$ ( $n=$ 790) of eligible and locatable controls. The nurses drew a blood sample and measured weight, height, and waist and hip circumferences at the time of interview. Age was based on age at diagnosis in cases or on age at selection in controls. Race was classified according to self-report. Fewer than $2 \%$ of the participants described themselves as races other than AfricanAmerican or white; these subjects were categorized with African-American women for statistical analysis. Women who used OCs or HRT for 3 months or longer were considered ever users. Use of HRT was evaluated among postmenopausal women.

Menopausal status was determined by information provided by participants in the interview. Women who were 50 years of age or older at the time of interview were considered to be postmenopausal if their periods had stopped naturally, due to surgery (hysterectomy and/or bilateral oophorectomy), or due to chemotherapy or radiation (unrelated to the present diagnosis of breast cancer). Women who were less than 50 years of age were considered postmenopausal if their periods stopped due to natural menopause, bilateral oophorectomy, radiation, or chemotherapy. All other women were classified as premenopausal.

\section{Statistical methods}

To quantify the associations between reproductive, hormonal, and other risk factors and breast cancer subtype defined by ESR1 908G mutation status, odds ratios (ORs) and 95\% confidence intervals (Cls) comparing each case subgroup to controls were calculated. The ORs for breast cancer were calculated using unconditional logistic regression as implemented in the SAS software program (version 8.2; SAS Institute Inc., Cary, NC, USA). Offset terms were incorporated into models using the SAS procedure, PROC GENMOD, to account for the sampling probabilities used to define eligible cases and controls [15]. Potential confounding was evaluated by including covariates in multivariate models, and these were selected based on their role in matching or their previously demonstrated relevance to breast cancer risk. These included age, race, menopausal status, family history of breast cancer in a first-degree relative, age at menarche, age at first full-term pregnancy (AFFTP) and parity incorporated into a composite variable (nulliparous, parity $\geq 1$ and $A F F T P=26$ years, parity $=1$ and AFFTP $<26$ years, parity $\geq 2$ /AFFTP $\geq 26$ years, and parity $\geq 2$ and AFFTP $<26$ years), lactation (ever, never), dura- tion of ovarian function defined as the number of years between onset of menarche and menopause (natural or otherwise), smoking, alcohol consumption (ever, never), and body mass index (BMI) (less than 25, 25 to less than 30, or greater than or equal to $30 \mathrm{~kg} / \mathrm{m}^{2}$ ). Ever smoking was defined as lifetime exposure to at least 100 cigarettes. Trend tests for risk factors with ordinal levels (for example, such as duration of $\mathrm{OC}$ use) were performed, and $P$ values were calculated using Wald $\chi^{2}$ statistics.

\section{Results \\ Characteristics of cases and controls}

The characteristics of cases and controls in the CBCS have been described previously [17]. Slightly more than half of the subjects were younger than 50 years of age, approximately half were premenopausal, and approximately $40 \%$ were African-American. Cases were significantly more likely than controls to have a first-degree family history of breast cancer.

For the present study, a total of 653 FFPE invasive breast tumors from cases in the CBCS were screened for mutations in a 104-base pair region of exon 4 surrounding codon 303 of ESR1. The clinical characteristics of cases evaluated for the ESR1 mutation have been reported previously [14]. The majority (88\%) of cases had stage 1 or 2 disease, $60 \%$ were node-negative, 59\% were ER-positive, and 79\% were diagnosed with invasive ductal carcinoma. Of the 653 breast cancer cases screened, 37 (5.7\%) were positive for the ESR1 A908G mutation. When analyzed according to ESR1 A908G mutation status, tumors that were mutation-positive were of somewhat larger size $(P=0.11)$, were of somewhat higher combined grade $(P=0.03$ grade II versus grade I), and were more likely to have mixed lobular/ductal histology $(P=0.10)$. However, there was no difference in ER protein expression between mutation-positive and mutation-negative breast tumors $(P=0.57)$.

\section{Risk factors for breast cancer according to ESR1 A908G mutation status}

Table 1 presents both minimally adjusted and more fully adjusted ORs and 95\% Cls comparing ESR1 mutation-positive cases $(n=37)$ and mutation-negative cases $(n=616)$ to controls $(n=790)$. Both mutation-positive and mutation-negative cases were less likely than controls to have ever breast fed, but this inverse association was more pronounced for mutation-positive cases ( $\mathrm{OR}=0.29,95 \% \mathrm{Cl}=0.12$ to 0.71 ) than for mutation-negative cases (OR $=0.69,95 \% \mathrm{Cl}=0.53$ to 0.89 ). Mutation-negative cases were more likely than controls to have younger age at menarche $(\mathrm{OR}=1.46,95 \% \mathrm{Cl}=$ 1.09 to 1.94$)$ and longer duration of ovarian function if postmenopausal $(\mathrm{OR}=1.82,95 \% \mathrm{Cl}=1.21$ to 2.74 for 36 years or more; $P_{\text {trend }}=0.004$ ). BMl overall was not associated with either mutation-positive or mutation-negative cancer; stratification on menopausal status yielded numbers of mutation-posi- 
Breast Cancer Research Vol 9 No 3 Conway et al.

Table 1

Associations between reproductive and other risk factors and breast cancer characterized by ESR1 A908G mutation status

\begin{tabular}{|c|c|c|c|c|c|c|c|c|c|c|c|c|c|c|}
\hline \multirow[t]{2}{*}{ Risk factor } & \multicolumn{2}{|c|}{$\begin{array}{l}\text { Controls } \\
(n=790)\end{array}$} & \multicolumn{2}{|c|}{$\begin{array}{l}\text { Mutation-positive } \\
\text { cases } \\
(n=37)\end{array}$} & \multirow[b]{2}{*}{ OR1a } & \multirow[b]{2}{*}{$95 \% \mathrm{Cl}$} & \multirow[b]{2}{*}{ OR2 ${ }^{b}$} & \multirow[b]{2}{*}{$95 \% \mathrm{Cl}$} & \multicolumn{2}{|c|}{$\begin{array}{l}\text { Mutation-negative } \\
\text { cases } \\
(n=616)\end{array}$} & \multirow[b]{2}{*}{ OR1 ${ }^{a}$} & \multirow[b]{2}{*}{$95 \% \mathrm{Cl}$} & \multirow[b]{2}{*}{ OR2b } & \multirow[b]{2}{*}{$95 \% \mathrm{Cl}$} \\
\hline & $n$ & $(\%)$ & $n$ & $(\%)$ & & & & & $n$ & $(\%)$ & & & & \\
\hline \multicolumn{15}{|l|}{ Age (years) } \\
\hline $50+$ & 383 & $(48.5)$ & 19 & $(51.4)$ & c & & c & & 246 & (39.9) & c & & c & \\
\hline$<50$ & 407 & $(51.5)$ & 18 & $(48.6)$ & & & & & 370 & $(60.1)$ & & & & \\
\hline \multicolumn{15}{|l|}{ Race } \\
\hline White & 458 & $(58.0)$ & 25 & $(67.6)$ & c & & c & & 371 & $(60.2)$ & c & & c & \\
\hline African-American & 332 & $(42.0)$ & 12 & (32.4) & & & & & 245 & (39.8) & & & & \\
\hline \multicolumn{15}{|l|}{ Menopausal status } \\
\hline Postmenopausal & 435 & $(55.1)$ & 22 & $(59.5)$ & 1.00 & --- & $1.00^{d}$ & --- & 295 & $(47.9)$ & 1.00 & --- & $1.00^{d}$ & --- \\
\hline Premenopausal & 355 & $(44.9)$ & 15 & $(40.5)$ & 1.27 & $0.40-4.05$ & 1.63 & $0.47-5.64$ & 321 & $(52.1)$ & 1.08 & $0.78-1.50$ & 1.12 & $0.80-1.58$ \\
\hline \multicolumn{15}{|c|}{$\begin{array}{l}\text { First-degree family history } \\
\text { of } B C\end{array}$} \\
\hline No & 671 & (88.3) & 26 & $(72.2)$ & 1.00 & --- & $1.00^{e}$ & --- & 523 & $(87.0)$ & 1.00 & --- & $1.00^{e}$ & --- \\
\hline Yes & 89 & $(11.7)$ & 10 & $(27.8)$ & 2.54 & $1.16-5.55$ & 2.69 & $1.15-6.28$ & 78 & $(13.0)$ & 1.17 & $0.84-1.62$ & 1.22 & $0.87-1.71$ \\
\hline \multicolumn{15}{|c|}{$\begin{array}{l}\text { First-degree family history } \\
\text { of } B C \text { and/or ovarian } \\
\text { cancer }\end{array}$} \\
\hline No & 664 & $(87.4)$ & 24 & $(66.7)$ & 1.00 & --- & $1.00^{e}$ & --- & 514 & $(85.5)$ & 1.00 & --- & $1.00^{e}$ & --- \\
\hline Yes & 96 & $(12.6)$ & 12 & (33.3) & 3.13 & $1.49-6.58$ & 3.48 & $1.56-7.76$ & 87 & (14.5) & 1.21 & $0.88-1.66$ & 1.27 & $0.92-1.76$ \\
\hline \multicolumn{15}{|c|}{ Prior benign breast biopsy } \\
\hline No & 649 & $(82.4)$ & 28 & $(80.0)$ & 1.00 & --- & 1.00 & --- & 518 & $(84.2)$ & 1.00 & --- & 1.00 & --- \\
\hline Yes & 139 & $(17.6)$ & 7 & $(20.0)$ & 1.21 & $0.51-2.87$ & 1.17 & $0.47-2.91$ & 97 & $(15.8)$ & 1.00 & $0.75-1.33$ & 0.94 & $0.70-1.28$ \\
\hline \multicolumn{15}{|l|}{ BMI } \\
\hline$<25$ & 250 & $(32.2)$ & 15 & $(41.7)$ & 1.00 & --- & $1.00^{f}$ & --- & 224 & $(37.1)$ & 1.00 & --- & $1.00^{f}$ & --- \\
\hline 25 to $<30$ & 250 & $(32.2)$ & 10 & $(27.8)$ & 0.73 & $0.32-1.69$ & 0.78 & $0.32-1.90$ & 180 & $(29.8)$ & 0.87 & $0.66-1.14$ & 0.83 & $0.63-1.10$ \\
\hline $30+$ & 277 & $(35.6)$ & 11 & $(30.5)$ & 0.80 & $0.34-1.89$ & 0.76 & $0.29-1.98$ & 200 & (33.1) & 0.85 & $0.64-1.12$ & 0.80 & $0.60-1.08$ \\
\hline \multicolumn{15}{|l|}{ Smoking } \\
\hline Never & 423 & (53.5) & 18 & $(48.7)$ & 1.00 & --- & $1.00 \mathrm{~g}$ & --- & 322 & $(52.3)$ & 1.00 & --- & $1.00 \mathrm{~g}$ & --- \\
\hline Ever & 367 & $(46.5)$ & 19 & (51.3) & 1.16 & $0.59-2.27$ & 1.04 & $0.49-2.19$ & 294 & $(47.7)$ & 1.08 & $0.87-1.34$ & 1.11 & $0.88-1.41$ \\
\hline \multicolumn{15}{|l|}{ Alcohol consumption } \\
\hline Never & 231 & (29.3) & 14 & (37.8) & 1.00 & -- & $1.00^{h}$ & --- & 178 & $(28.9)$ & 1.00 & --- & $1.00^{\mathrm{h}}$ & --- \\
\hline Ever & 558 & $(70.7)$ & 23 & $(62.2)$ & 0.79 & $0.39-1.63$ & 0.74 & $0.32-1.71$ & 438 & $(71.1)$ & 0.95 & $0.75-1.22$ & 0.88 & $0.67-1.16$ \\
\hline Age at menarche (ye & & & & & & & & & & & & & & \\
\hline $14+$ & 203 & $(25.8)$ & 10 & $(27.0)$ & 1.00 & --- & $1.00^{\mathrm{i}}$ & --- & 130 & $(21.1)$ & 1.00 & -- & $1.00^{i}$ & --- \\
\hline 13 & 206 & $(26.2)$ & 9 & (24.3) & 0.96 & $0.38-2.44$ & 1.22 & $0.43-3.41$ & 165 & $(26.8)$ & 1.28 & $0.95-1.75$ & 1.38 & $1.01-1.91$ \\
\hline$\leq 12$ & 378 & $(48.0)$ & 18 & $(48.6)$ & 1.12 & $0.50-2.50$ & 1.42 & $0.57-3.53$ & 321 & $(52.1)$ & 1.36 & $1.04-1.79$ & 1.46 & $1.09-1.94$ \\
\hline Parity & & & & & & & & & & & & & & \\
\hline Nulliparous & 89 & $(11.3)$ & 5 & $(13.5)$ & 1.00 & -- & $1.00 \mathrm{j}$ & -- & 100 & $(16.2)$ & 1.00 & -- & $1.00 \mathrm{j}$ & -- \\
\hline Parous & 701 & $(88.7)$ & 32 & $(86.5)$ & 0.93 & $0.34-2.54$ & 1.30 & $0.44-3.85$ & 516 & $(83.8)$ & 0.76 & $0.55-1.04$ & 0.92 & $0.65-1.29$ \\
\hline $\begin{array}{l}\text { Age at first full-term } \\
\text { pregnancy (years) }\end{array}$ & & & & & & & & & & & & & & \\
\hline Nulliparous & 89 & (11.3) & 5 & (13.9) & 1.00 & --. & $1.00 \mathrm{j}$ & --- & 100 & $(16.3)$ & 1.00 & --- & $1.00 j$ & --- \\
\hline $10-19$ & 236 & $(29.9)$ & 8 & $(22.2)$ & 0.74 & $0.23-2.39$ & 1.03 & $0.28-3.79$ & 181 & $(29.5)$ & 0.82 & $0.57-1.16$ & 0.99 & $0.67-1.46$ \\
\hline $20-24$ & 265 & $(33.6)$ & 11 & $(30.5)$ & 0.82 & $0.27-2.52$ & 1.14 & $0.35-3.74$ & 181 & $(29.5)$ & 0.73 & $0.51-1.03$ & 0.88 & $0.60-1.27$ \\
\hline
\end{tabular}


Table 1 (Continued)

\begin{tabular}{|c|c|c|c|c|c|c|c|c|c|c|c|c|c|c|}
\hline $25+$ & 198 & $(25.2)$ & 12 & (33.3) & 1.20 & $0.40-3.63$ & 1.71 & $0.51-5.78$ & 152 & $(24.8)$ & 0.73 & $0.51-1.05$ & 0.90 & $0.60-1.34$ \\
\hline \multicolumn{15}{|c|}{ Number full-term births } \\
\hline Nulliparous & 89 & $(11.3)$ & 5 & (13.5) & 1.00 & --- & $1.00 \mathrm{j}$ & --- & 100 & $(16.2)$ & 1.00 & --- & $1.00 \mathrm{j}$ & --- \\
\hline 1 & 141 & $(17.8)$ & 5 & (13.5) & 0.74 & $0.20-2.69$ & 0.99 & $0.26-3.78$ & 117 & $(19.0)$ & 0.79 & $0.54-1.17$ & 0.89 & $0.59-1.34$ \\
\hline 2 or more & 560 & $(70.9)$ & 27 & $(73.0)$ & 0.98 & $0.36-2.72$ & 1.43 & $0.47-4.35$ & 399 & $(64.8)$ & 0.75 & $0.54-1.03$ & 0.92 & $0.65-1.31$ \\
\hline \multicolumn{15}{|l|}{ Breastfeeding } \\
\hline Never & 476 & $(60.3)$ & 26 & (70.3) & 1.00 & -- & $1.00^{k}$ & --- & 413 & $(67.0)$ & 1.00 & --- & $1.00^{k}$ & --- \\
\hline Ever & 314 & (39.7) & 11 & $(29.7)$ & 0.52 & $0.25-1.09$ & 0.29 & $0.12-0.71$ & 203 & (33.0) & 0.70 & $0.56-0.87$ & 0.69 & $0.53-0.89$ \\
\hline \multicolumn{15}{|c|}{$\begin{array}{l}\text { Age at menopause (years) } \\
\text { (postmenopausal) }\end{array}$} \\
\hline$<45$ & 192 & $(45.6)$ & 10 & $(45.5)$ & 1.00 & - & $1.00^{d}$ & --- & 117 & (39.9) & 1.00 & --- & $1.00^{d}$ & --- \\
\hline $45-49$ & 119 & $(28.3)$ & 7 & (31.8) & 1.13 & $0.42-3.08$ & 1.47 & $0.45-4.79$ & 93 & $(31.7)$ & 1.38 & $0.96-1.97$ & 1.39 & $0.95-2.03$ \\
\hline $50+$ & 110 & $(26.1)$ & 5 & $(22.7)$ & 0.82 & $0.27-2.53$ & 1.32 & $0.36-4.77$ & 83 & $(28.3)$ & 1.39 & $0.95-2.03$ & 1.50 & $1.00-2.24$ \\
\hline \multicolumn{15}{|c|}{$\begin{array}{l}\text { Duration of ovarian } \\
\text { function (years) } \\
\text { (postmenopausal) }\end{array}$} \\
\hline$\leq 29$ & 139 & (33.2) & 6 & $(27.2)$ & 1.00 & --- & $1.00^{d}$ & --- & 79 & $(27.0)$ & 1.00 & --- & $1.00^{d}$ & --- \\
\hline $30-35$ & 141 & (33.6) & 10 & $(45.4)$ & 1.66 & $0.58-4.74$ & 1.78 & $0.49-6.46$ & 98 & (33.4) & 1.33 & $0.90-1.95$ & 1.32 & $0.88-1.99$ \\
\hline $36+$ & 139 & (33.2) & 6 & $(27.2)$ & 0.98 & $0.30-3.18$ & 1.38 & $0.35-5.45$ & 116 & (39.6) & 1.69 & $1.15-2.48$ & 1.82 & $1.21-2.74$ \\
\hline$P_{\text {trend }}$ & & & & & & 0.97 & & 0.70 & & & & 0.007 & & 0.004 \\
\hline
\end{tabular}

aOR1 minimally adjusted for age, race, and offsets.

bOR2 adjusted for age, race, offsets, menopausal status (except d), family history of breast cancer (except e), BMI $(<25,25$ to $<30,30+)$ (except $\mathrm{f})$, smoking status (never, former, current) (except g), alcohol intake (ever, never) (except h), age at menarche $(<11,11-12,13,14+)($ except i), parity/age first full-term pregnancy (except j), and breast feeding (never, ever) (except k).

cORs were not calculated for age or race as these factors were part of the sampling scheme. BC, breast cancer; BMI, body mass index; Cl, confidence interval; ESR1, estrogen receptor alpha; OR, odds ratio.

tive cases that were too sparse to analyze. Adjustment for additional covariates did not appreciably alter the ORs.

Compared with controls, ESR1 A908G mutation-positive cases were significantly more likely to have a first-degree family history of breast cancer $(\mathrm{OR}=2.69,95 \% \mathrm{Cl}=1.15$ to 6.28 ) or a first-degree family history of breast and/or ovarian cancer (OR $=3.48,95 \% \mathrm{Cl}=1.56$ to 7.76 ), whereas mutation-negative cases did not $(\mathrm{OR}=1.22,95 \% \mathrm{Cl}=0.87$ to 1.71 for first-degree family history of breast cancer). Mutationpositive cases were also directly compared to the mutationnegative cases to determine the degree of risk heterogeneity between these two disease subtypes. First-degree family histories of breast cancer ( $\mathrm{OR}=2.65,95 \% \mathrm{Cl}=1.15$ to 6.09 ) or of breast and/or ovarian cancer (OR $=3.15,95 \% \mathrm{Cl}=1.44$ to 6.91) were both significantly associated with ESR1 A908G mutation positivity (not shown).

As shown in Table 2, multivariate logistic regression analyses indicated that ESR1 mutation-positive breast cancer cases were more likely to have ever used OCs $(\mathrm{OR}=1.72,95 \% \mathrm{Cl}$ $=0.66$ to 4.44 ), but this result was not statistically significant. An association with $\mathrm{OC}$ use was strongest among long-term users $(\mathrm{OR}=3.73,95 \% \mathrm{Cl}=1.16$ to 12.03 for duration more than 10 years) and recent users (OR $=3.63,95 \% \mathrm{Cl}=0.80$ to 16.45 for use within 10 years). Women who had used OCs for more than 10 years and within the past 10 years showed the greatest risk of ESR1 mutation-positive breast cancer (OR $=6.49,95 \% \mathrm{Cl}=1.32$ to 31.89 ).

Several ORs for OC use were elevated but were not statistically significant, probably due to the small number of mutationpositive tumors. The $P$ values for trend among ESR1 A908G mutation-positive cases were 0.02 for duration of $\mathrm{OC}$ use (less than 5,5 to 10 , or more than 10 years) and 0.10 for recency of $\mathrm{OC}$ use (10 or more years or less than 10 years). In contrast to our findings in ESR1 mutation-positive cases, OC use, even long-term or recent use, was not associated with mutation-negative breast cancer. Among mutation-negative cases, the $P_{\text {trend }}$ values were 0.84 for duration of $\mathrm{OC}$ use (less than 5,5 to 10 , or more than 10 years) and 0.56 for years since stopping (10 or more years or less than 10 years). Other measures of OC use similarly showed no associations with ESR1 mutation-negative breast cancer.

Comparing mutation-positive to mutation-negative cases, $\mathrm{OC}$ use for more than 10 years and $O C$ use within the past 10 years were both associated with ESR1 A908G mutation positivity, but these ORs were not statistically significant (OR $=2.66,95 \% \mathrm{Cl}=0.81$ to 8.67 for use for more than 10 years; $\mathrm{OR}=3.65,95 \% \mathrm{Cl}=0.86$ to 15.47 for use within the past 10 
Table 2

Associations between OC or HRT use and breast cancer defined by ESR1 A908G mutation status

\begin{tabular}{|c|c|c|c|c|c|c|c|c|c|c|c|c|}
\hline \multirow[t]{2}{*}{ Risk factor } & \multicolumn{2}{|c|}{$\begin{array}{l}\text { Controls } \\
(n=790)\end{array}$} & \multicolumn{2}{|c|}{$\begin{array}{l}\text { Mutation-positive } \\
\text { cases }(n=37)\end{array}$} & \multicolumn{2}{|c|}{$\begin{array}{l}\text { Mutation-positive } \\
\text { cases vs. controls }\end{array}$} & \multicolumn{2}{|c|}{$\begin{array}{l}\text { Mutation-negative } \\
\text { cases }(n=616)\end{array}$} & \multicolumn{2}{|c|}{$\begin{array}{l}\text { Mutation-negative } \\
\text { cases vs. controls }\end{array}$} & \multicolumn{2}{|c|}{$\begin{array}{c}\text { Mutation-positive } \\
\text { vs. mutation-negative } \\
\text { cases }\end{array}$} \\
\hline & $n$ & (\%) & $n$ & (\%) & $\mathrm{OR}^{\mathrm{a}}$ & $95 \% \mathrm{Cl}$ & $n$ & $(\%)$ & $\mathrm{OR}^{\mathrm{a}}$ & $95 \% \mathrm{Cl}$ & $\mathrm{OR}^{\mathrm{a}}$ & $95 \% \mathrm{Cl}$ \\
\hline \multicolumn{13}{|l|}{ OC use } \\
\hline Never & 319 & $(40.4)$ & 14 & (37.8) & 1.00 & --- & 224 & $(36.5)$ & 1.00 & --- & 1.00 & -- \\
\hline Ever & 470 & $(59.6)$ & 23 & $(62.2)$ & 1.72 & $0.66-4.44$ & 390 & $(63.5)$ & 1.15 & $0.87-1.52$ & 1.53 & $0.61-3.84$ \\
\hline \multicolumn{13}{|l|}{ OC duration } \\
\hline Never & 319 & $(40.4)$ & 14 & (37.8) & 1.00 & -- & 224 & (36.5) & 1.00 & -- & 1.00 & -- \\
\hline$<5$ years & 232 & $(29.4)$ & 8 & $(21.6)$ & 1.08 & $0.35-3.38$ & 194 & (31.6) & 1.27 & $0.94-1.73$ & 1.01 & $0.33-3.06$ \\
\hline $5-10$ years & 162 & $(20.5)$ & 8 & $(21.6)$ & 1.75 & $0.53-5.77$ & 123 & $(20.0)$ & 0.95 & $0.67-1.35$ & 1.79 & $0.59-5.43$ \\
\hline$>10$ years & 76 & (9.6) & 7 & (8.9) & 3.73 & $1.16-12.03$ & 73 & (11.9) & 1.18 & $0.77-1.81$ & 2.66 & $0.81-8.67$ \\
\hline$P_{\text {trend }}$ & & & & & & 0.02 & & & & 0.84 & & 0.07 \\
\hline \multicolumn{13}{|c|}{ Age at first use of OCs } \\
\hline Never & 319 & $(40.4)$ & 14 & (37.8) & 1.00 & --- & 224 & $(36.6)$ & 1.00 & --- & 1.00 & --- \\
\hline$\leq 20$ years & 215 & $(27.2)$ & 8 & $(21.6)$ & 1.18 & $0.32-4.42$ & 192 & $(31.4)$ & 0.99 & $0.69-1.41$ & 1.44 & $0.41-5.09$ \\
\hline$>20$ years & 255 & (32.3) & 15 & $(40.5)$ & 1.81 & $0.71-4.63$ & 196 & $(32.0)$ & 1.20 & $0.89-1.60$ & 1.56 & $0.61-3.96$ \\
\hline \multicolumn{13}{|c|}{ Age at last use of OCs } \\
\hline Never & 319 & $(40.4)$ & 14 & $(37.8)$ & 1.00 & -- & 224 & $(36.6)$ & 1.00 & -- & 1.00 & -- \\
\hline$\leq 30$ years & 273 & $(34.6)$ & 10 & $(27.0)$ & 1.18 & $0.36-3.90$ & 226 & $(36.9)$ & 1.10 & $0.80-1.52$ & 1.39 & $0.45-4.25$ \\
\hline$>30$ years & 197 & $(25.0)$ & 13 & $(35.1)$ & 1.97 & $0.75-5.15$ & 162 & $(26.5)$ & 1.16 & $0.85-1.58$ & 1.63 & $0.62-4.29$ \\
\hline \multicolumn{13}{|c|}{ Year of first $O C$ use } \\
\hline Never & 319 & $(40.4)$ & 14 & $(37.8)$ & 1.00 & -- & 224 & $(36.6)$ & 1.00 & --- & 1.00 & -- \\
\hline$\leq 1975$ & 389 & $(49.3)$ & 19 & $(51.4)$ & 1.72 & $0.67-4.43$ & 292 & $(47.7)$ & 1.15 & $0.87-1.52$ & 1.54 & $0.61-3.89$ \\
\hline$>1975$ & 81 & $(10.3)$ & 4 & $(10.8)$ & 1.51 & $0.28-8.12$ & 96 & $(15.7)$ & 0.98 & $0.62-1.55$ & 1.58 & $0.35-7.13$ \\
\hline \multicolumn{13}{|c|}{ First use before first full-term pregnancy } \\
\hline No & 233 & $(54.8)$ & 11 & $(57.9)$ & 1.00 & --- & 169 & $(51.1)$ & 1.00 & --- & 1.00 & --- \\
\hline Yes & 192 & $(45.2)$ & 8 & $(42.1)$ & 0.66 & $0.15-2.93$ & 162 & $(48.9)$ & 1.12 & $0.76-1.66$ & 0.78 & $0.18-3.31$ \\
\hline \multicolumn{13}{|c|}{ Recency of $O C$ use (years since stopping) } \\
\hline Never & 319 & $(42.3)$ & 14 & $(37.8)$ & 1.00 & --- & 224 & $(39.4)$ & 1.00 & --- & 1.00 & --- \\
\hline $10+$ & 374 & $(49.5)$ & 17 & $(45.9)$ & 1.70 & $0.65-4.43$ & 278 & $(48.8)$ & 1.15 & $0.86-1.52$ & 1.52 & $0.59-3.92$ \\
\hline$<10$ & 62 & (8.2) & 6 & $(16.2)$ & 3.63 & $0.80-16.45$ & 67 & $(11.8)$ & 1.06 & $0.65-1.72$ & 3.65 & $0.86-15.47$ \\
\hline $\mathrm{P}_{\text {trend }}$ & & & & & & 0.10 & & & & 0.56 & & 0.10 \\
\hline \multicolumn{13}{|c|}{$\mathrm{OC}$ duration and recency of use (years since stopping) } \\
\hline Never & 319 & $(42.3)$ & 14 & $(37.8)$ & 1.00 & --- & 224 & $(39.4)$ & 1.00 & -- & 1.00 & -- \\
\hline$<10,<10$ & 31 & $(4.1)$ & 2 & $(5.4)$ & 1.43 & $0.19-10.77$ & 32 & $(5.6)$ & 0.84 & $0.44-1.58$ & 3.04 & $0.46-20.06$ \\
\hline$<10,10+$ & 315 & $(41.7)$ & 13 & $(35.1)$ & 1.53 & $0.55-4.26$ & 245 & $(43.1)$ & 1.21 & $0.90-1.62$ & 1.25 & $0.46-3.41$ \\
\hline $10+,<10$ & 31 & $(4.1$ & 4 & $(10.8)$ & 6.49 & $1.32-31.89$ & 35 & $(6.2)$ & 1.32 & $0.73-2.38$ & 3.53 & $0.73-17.09$ \\
\hline $10+, 10+$ & 59 & (7.8) & 4 & $(10.8)$ & 1.93 & $0.52-7.11$ & 33 & $(5.8)$ & 0.81 & $0.49-1.35$ & 3.01 & $0.78-11.59$ \\
\hline \multicolumn{13}{|c|}{ Postmenopausal HRT use } \\
\hline Never & 219 & $(50.3)$ & 13 & $(59.1)$ & 1.00 & --- & 180 & $(61.0)$ & 1.00 & --- & 1.00 & --- \\
\hline Ever & 216 & $(49.7)$ & 9 & $(40.9)$ & 0.83 & $0.28-2.42$ & 115 & $(39.0)$ & 0.62 & $0.44-0.87$ & 1.23 & $0.42-3.61$ \\
\hline \multicolumn{13}{|c|}{ Postmenopausal HRT duration } \\
\hline Never & 219 & $(50.3)$ & 13 & $(59.1)$ & 1.00 & -- & 180 & $(61.0)$ & 1.00 & --- & 1.00 & -- \\
\hline$<5$ years & 105 & $(24.1)$ & 3 & $(13.6)$ & 0.60 & $0.14-2.51$ & 56 & $(19.0)$ & 0.61 & $0.40-0.92$ & 1.37 & $0.30-6.24$ \\
\hline $5+$ years & 111 & $(25.6)$ & 6 & $(27.3)$ & 1.07 & $0.31-3.70$ & 59 & $(20.0)$ & 0.64 & $0.42-0.96$ & 1.17 & $0.35-3.91$ \\
\hline
\end{tabular}

aORs adjusted for sampling fraction, age, race, menopausal status (where appropriate), first-degree family history of breast cancer, parity/age first full-term pregnancy, breast feeding (ever, never), smoking status (never, former, current), alcohol intake (ever, never), age at menarche $(<11,11-$ $12,13,14+)$, and body mass index $(<25,25$ to $<30,30+)$. $\mathrm{Cl}$, confidence interval; ESR1, estrogen receptor alpha; HRT, hormone replacement therapy; OC, oral contraceptive; OR, odds ratio. 
years). ORs were suggestive of a positive trend, but the trend tests were not statistically significant.

Among postmenopausal women, there was no clear evidence for an association of HRT use with ESR1 A908G mutationpositive cancer (OR $=0.83,95 \% \mathrm{Cl}=0.28$ to 2.42 ), but HRT use was inversely associated with mutation-negative breast cancer $(\mathrm{OR}=0.62,95 \% \mathrm{Cl}=0.44$ to 0.87 for ever users; OR $=0.64,95 \% \mathrm{Cl}=0.42$ to 0.96 for duration of 5 or more years) (Table 2). We were unable to analyze HRT according to former and current use because the numbers in each case group were too sparse.

First-degree family history of breast cancer was associated with ESR1 mutation-positive breast cancer (OR $=2.54,95 \%$ $\mathrm{Cl}=1.16$ to 5.55 ) (Table 1 ). Further adjustment for OC use had little effect on this association $(\mathrm{OR}=2.71,95 \% \mathrm{Cl}=1.16$ to 6.33). These results suggest that $O C$ use and family history of breast cancer may independently influence risk of the ESR1 mutation-positive subset of breast cancer.

\section{Discussion}

Evidence is accumulating to suggest that breast cancer is a collection of biologically distinct disease subtypes characterized by unique gene expression profiles, molecular or protein markers, and that exhibit variable clinical behavior, prognosis, and response to therapies [9,10,18-21]. Similarly, data obtained from some epidemiologic studies of breast cancer suggest that tumor subsets classified according to certain somatic or protein expression changes may be associated with specific etiologic risk factors [17,22-26]. Consistent with this, our study has revealed that first-degree family history of breast cancer (or breast and/or ovarian cancer) may be a risk factor for breast tumors carrying the ESR1 A908G mutation. Recent (within 10 years) and long-term (more than 10 years) use of OCs may also be associated with mutation-positive breast cancer; however, due to small numbers, most of these results were not statistically significant. In contrast, mutationnegative breast cancer was not associated with $\mathrm{OC}$ use but instead was associated with several reproductive factors, including longer duration of ovarian function and younger age at menarche, which increase exposure to endogenous hormones. HRT use was inversely correlated with the mutationnegative breast cancer subtype, but due to the small number of postmenopausal cases carrying the mutation, an effect of HRT on ESR1 A908G mutation-positive breast cancer could not be adequately assessed.

Many hormonal or reproductive factors that increase exposure to estrogen and/or progesterone are risk factors for breast cancer [1], and the contribution of OC use to breast cancer development has been extensively researched. Recent and longer durations of $\mathrm{OC}$ use have shown the most consistently positive, though small, associations with breast cancer [2735], observations which are compatible with our findings for the ESR1 A908G mutation-positive cases. The 1996 metaanalysis of the published data on breast cancer risk and hormonal contraceptives by the Collaborative Committee on Hormonal Factors in Breast Cancer, the most comprehensive assessment of the impact of OCs on breast cancer risk [30], found that current and recent users of combined OCs had a small increased risk of breast cancer which decreased with time such that no significant excess risk remained 10 years after last use. Breast tumors that developed among ever OC users were less likely to have distant metastases; however, other characteristics of the primary tumor were not specifically evaluated. Young age at first use of OCs and use before the first full-term pregnancy carry elevated risks for breast cancer in several studies $[28,32,35]$; however, we found only a small, non-significant association with later age at first use of OCs among the ESR1 A908G mutation-positive subgroup. Additionally, associations with OCs have been noted among some subsets of breast cancer cases, including younger women $[27,28,35]$, those with a family history of breast cancer [36], germline mutations in BRCA1 or BRCA2 [37-39], or other genetic polymorphisms [40]. Case subgroups defined by certain tumor characteristics have been associated with $O C$ use, including lobular or mixed lobular/ductal histology [41], and those expressing ER [26], HER2 [17], p53 [23], or cyclin D1 [24].

In our study, ESR1 A908G mutation-positive breast cancer was strongly associated with recent and long-term $\mathrm{OC}$ use even though there was only a small main effect of $O C$ use in the CBCS [31]. This finding suggests that characterization of breast tumors according to ESR1 A908G mutation status may help to uncover a subgroup of women for whom OC use may be a stronger risk factor. Furthermore, the mutation-negative subgroup may be associated with certain risk factors that represent greater exposure to endogenous hormones. Li and colleagues [42] noted distinct effects of exogenous versus endogenous hormonal factors on tumor histology, with OC use being more strongly associated with the development of lobular and mixed lobular/ductal breast tumors whereas endogenous hormonal factors, including longer duration of ovarian function and higher BMI, were more strongly associated with risk of ductal breast cancer. Several other case-control studies have also found that use of combined HRT or OC was associated mainly with an increase in lobular and mixed lobular/ductal breast cancer $[41,43,44]$. The relationship between ESR1 A908G mutation-positive breast cancer and $\mathrm{OC}$ use is not surprising as the mutation-positive subgroup of tumors in the CBCS was more likely to have mixed lobular/ ductal histology [14].

These results suggest the possibility that endogenous and exogenous hormones may be differentially associated with development of breast cancer characterized by the presence or absence of the ESR1 A908G mutation. The exact basis for this is unclear but could be related to qualitative and/or quan- 
titative differences between contraceptive hormones and estradiol (and/or progesterone), the timing of exposure to OCs, or differences in the biological effects elicited by the mutant and wild-type receptors.

Exposure to OCs, particularly the estrogen component, increases proliferative activity in breast epithelium [45-50]. The ESR1 A908G mutant was reported to be hypersensitive to estrogen and was associated with cellular proliferation at sub-physiologic levels of estradiol in vitro compared with the wild-type receptor [11]. Additionally, the A908G mutation was originally detected in breast hyperplasias [11], which may be risk factors and, in some instances, early precursors of invasive breast cancer [51-53]. We have also detected this mutation in benign breast lesions that contain histologic features other than usual hyperplasia (K. Conway, unpublished data). Given the abnormal function described for this somatic ESR1 A908G variant and its presence in very early breast lesions, it is biologically plausible that exposure of breast tissue to exogenous hormones during the premenopausal years could be associated with the subgroup of breast tumors carrying this mutation. From a mechanistic standpoint, our results suggest that OCs could interact with the pre-existing ESR1 A908G mutant receptor in early pre-neoplastic breast lesions to stimulate epithelial proliferation, thus driving the accumulation of additional genetic errors leading to neoplasia. The possibility that such a common exposure as OC use might stimulate the outgrowth of cells carrying this somatic mutation makes the ESR1 A908G variant a potentially important candidate marker for studies of breast cancer etiology and progression.

Studies on combined estrogen and progestin HRT have generally found longer duration and recent use of HRT to be associated with increased risk of breast cancer [54]. HRT with estrogen alone or combined estrogen and progestin is associated with increased proliferation in normal breast tissue of postmenopausal women [55]. Postmenopausal HRT use was somewhat protective for the mutation-negative subgroup, similar to what was previously reported overall in the CBCS [56].

Compared with controls, breast cancer cases in the CBCS with ESR1 A908G mutation-positive tumors were more likely to have a first-degree family history of breast cancer whereas the mutation-negative cases were not; this finding was supported by case-case comparisons. This association is unlikely to be related to germline defects in BRCA1 or BRCA2 since 21 of the 37 ESR1 mutation-positive cases had previously been screened for mutations in these genes but none was found [57] (B Newman and M-C King, unpublished data). However, we cannot rule out the possibility that these cases may carry germline variations in other genes which could influence breast cancer susceptibility and which include variants within ESR1 that may be linked with mutation status. Several previous studies have evaluated the risk of breast cancer associated with $\mathrm{OC}$ use among women with a family history of breast cancer $[33,40,58]$ or those carrying germline defects in BRCA1 or BRCA2 [37-39]. Grabrick and colleagues [36] suggested that $\mathrm{OC}$ use was more strongly associated with breast cancer among women with a family history, but at least two subsequent studies have not supported this finding $[29,58]$. However, in some studies, OC use has been found to increase breast cancer risk among women with known $B R C A 1$ or BRCA2 germline variants [37-39].

The primary strengths of this study are the population-based case series comprised mainly of early stage tumors, the careful assessment of the ESR1 A908G mutation by means of a stringent screening algorithm, and the large sample size nearly half of which consisted of premenopausal women. Despite the large number of cases evaluated $(n=653)$, this study is limited by the small number of mutation-positive breast tumors identified. Therefore, our results should be interpreted with caution. However, the significant findings on OC use and ESR1 A908G mutation-positive breast cancer warrant further study in larger data sets.

\section{Conclusion}

Characterization of breast tumors for the ESR1 A908G point mutation, shown by Fuqua and colleagues [9] to be hypersensitive to estrogen, may reveal important etiologic clues. ESR1 A908G mutation-positive breast cancer was significantly associated with longer duration and recent use of OCs and with a first-degree family history of breast cancer, suggesting that OCs may interact with the ESR1 A908G mutant receptor in the development of some breast tumors. Some reproductive factors linked to greater exposure to endogenous hormones, including younger age at menarche and longer duration of ovarian function, were associated with the mutation-negative subgroup, suggesting that endogenous hormonal factors may be more important for mutation-negative cancer. Additional studies will be required to confirm these findings.

\section{Competing interests}

The authors declare that they have no competing interests.

\section{Authors' contributions}

EP and DT conducted the laboratory analyses. C-KT conducted the statistical analyses. KC, RCM, BN, and PM participated in the interpretation of results and writing of the manuscript. SNE conducted the laboratory analyses and participated in the interpretation of results and writing of the manuscript. All authors read and approved the final manuscript.

\section{Acknowledgements}

This work was supported by the University of North Carolina Breast Cancer SPORE (Specialized Program of Research Excellence) grant no. CA58223 from the National Cancer Institute. We also thank the staff and participants of the CBCS for their invaluable contributions to the study. 


\section{References}

1. Pike MC, Spicer DV, Dahmoush L, Press MF: Estrogens, progestogens, normal breast cell proliferation, and breast cancer risk. Epidemiol Rev 1993, 15:17-35.

2. Murphy LC, Dotzlaw H, Leygue E, Douglas D, Coutts A, Watson $\mathrm{PH}$ : Estrogen receptor variants and mutations. J Steroid Biochem Molec Biol 1997, 62:363-372.

3. Herynk MH, Fuqua SA: Estrogen receptor mutations in human disease. Endocr Rev 2004, 25:869-898.

4. Dotzlaw H, Leygue E, Watson PH, Murphy LC: Expression of estrogen receptor-beta in human breast tumors. J Clin Endocrinol Metab 1997, 82:2371-2374.

5. Enmark E, Pelto-Huikko M, Grandien K, Lagercrantz S, Lagercrantz J, Fried G, Nordenskjold M, Gustafsson JA: Human estrogen receptor $\beta$-gene structure, chromosomal localization, and expression pattern. J Clin Endocrinol Metab 1997, 82:4258-4265.

6. Hu YF, Lau KM, Ho S-M, Russo J: Increased expression of estrogen receptor in chemically transformed human breast epithelial cells. Int $J$ Oncol 1998, 12:1225-1228.

7. Iwao K, Miyoshi Y, Egawa C, Ikeda N, Noguchi S: Quantitative analysis of estrogen receptor-beta mRNA and its variants in human breast cancer. Int J Cancer 2000, 88:733-736.

8. Henderson BE, Feigelson HS: Hormonal carcinogenesis. Carcinogenesis 2000, 21:427-433.

9. Fuqua SA, Wiltschke C, Zhang OX, Borg A, Castles CG, Friedrichs WE, Hopp T, Hilsenbeck S, Mohsin S, O'Connell P, et al.: A hypersensitive estrogen receptor- $\alpha$ mutation in premalignant breast lesions. Cancer Res 2000, 60:4026-4029.

10. Zubairy S, Cui Y, Fuqua SA: The K303R estrogen receptor alpha breast cancer mutant generates a new Akt kinase site. Proc Am Assoc Cancer Res 2004, 45:659.

11. Cui $Y$, Zhang M, Pestell R, Curran EM, Welshons WV, Fuqua SAW: Phosphorylation of estrogen receptor $\alpha$ blocks its acetylation and regulates estrogen sensitivity. Cancer Res 2004, 64:9199-9208.

12. Conway K, Parrish E, Edmiston SN, Tolbert D, Tse J, Geradts J, Livasy $\mathrm{C}$, Singh $\mathrm{H}$, Newman B, Millikan RC: The estrogen receptor alpha A908G (K303R) mutation occurs at low frequency in invasive breast tumors: results of a population-based study. Breast Cancer Res 2005, 7:R871-880.

13. Perou CM, Sorlie T, Eisen MB, van de Rijn M, Jeffrey SS, Rees CA, Pollack JR, Ross DT, Johnsen H, Akslen LA, et al:: Molecular portraits of human breast tumors. Nature 2000, 406:747-752.

14. Sorlie T, Perou CM, Tibshirani R, Aas T, Geisler S, Johnsen H, Hastie T, Eisen MB, van de Rijn M, Jeffrey SS, et al:: Gene expression patterns of breast carcinomas distinguish tumor subclasses with clinical implications. Proc Natl Acad Sci USA 2001, 98:10869-10874

15. Newman B, Moorman P, Millikan R, Qaqish BF, Geradts J, Aldrich TE, Liu ET: The Carolina Breast Cancer Study: integrating population-based epidemiology and molecular biology. Breast Cancer Res Treat 1995, 35:51-60.

16. Dressler LG, Geradts J, Burroughs M, Cowan D, Millikan RC, Newman B: Policy guidelines for the utilization of formalin-fixed, paraffin-embedded tissue sections: the UNC SPORE experience. Breast Cancer Res Treat 1999, 58:31-39.

17. Huang WY, Newman B, Millikan RC, Schell MJ, Hulka BS, Moorman PG: Hormone-related factors and risk of breast cancer in relation to estrogen receptor and progesterone receptor status. Am J Epidemiol 2000, 151:703-714.

18. Rouzier R, Perou CM, Symmans WF, Ibrahim N, Cristofanilli M, Anderson $\mathrm{K}$, Hess KR, Stec J, Ayers M, Wagner $\mathrm{P}$, et al.: Breast cancer molecular subtypes respond differently to preoperative chemotherapy. Clin Cancer Res 2005, 11:5678-5685.

19. Huang E, Cheng SH, Dressman H, Pittman J, Tsou MH, Horng CF, Bild A, Iversen ES, Liao M, Chen CM, et al:: Gene expression predictors of breast cancer outcomes. Lancet 2003, 361:1590-1596.

20. Carey LA, Perou CM, Livasy CA, Dressler LG, Cowan D, Conway $\mathrm{K}$, Karaca G, Troester MA, Tse CK, Edmiston S, et al:: Race, breast cancer subtypes, and survival in the Carolina Breast Cancer Study. JAMA 2006, 295:2492-2502.

21. Oh DS, Troester MA, Usary J, Hu Z, He X, Fan C, Wu J, Carey LA, Perou CM: Estrogen-regulated genes predict survival in hor- mone receptor-positive breast cancers. J Clin Oncol 2006, 24:1656-1664.

22. Conway K, Edmiston SN, Cui L, Drouin SS, Pang J, He M, Tse CK Geradts J, Dressler L, Liu ET, et al.: Prevalence and spectrum of p53 mutations associated with smoking in breast cancer. Cancer Res 2002, 62:1987-1995.

23. Furberg H, Millikan RC, Geradts J, Gammon MD, Dressler LG Ambrosone CB, Newman B: Reproductive factors in relation to breast cancer characterized by $\mathrm{p} 53$ protein expression. Cancer Causes Contro/ 2003, 14:609-618.

24. Terry MB, Gammon MD, Schoenberg JB, Brinton LA, Arber N, Hibshoosh $\mathrm{H}$ : Oral contraceptive use and cyclin D1 overexpression in breast cancer among young women. Cancer Epidemiol Biomarkers Prev 2002, 11:1100-1103.

25. Tsakountakis N, Sanidas E, Stathopoulos E, Kafousi M, Anogiannaki N, Georgoulias V, Tsiftsis DD: Correlation of breast cancer risk factors with HER-2/neu protein overexpression according to menopausal and estrogen receptor status. BMC Womens Health 2005, 5:1-9.

26. Potter JD, Cerhan JR, Sellers TA, McGovern PG, Drinkard C, Kushi LR, Folsom AR: Progesterone and estrogen receptors and mammary neoplasia in the lowa Women's Health Study: how many kinds of breast cancer are there? Cancer Epidemiol Biomarkers Prev 1995, 4:319-326.

27. Althuis MD, Brogan DR, Coates RJ, Daling JR, Gammon MD, Malone KE, Schoenberg JB, Brinton LA: Breast cancers among very young premenopausal women. Cancer Causes Control 2003, 14:151-160.

28. Althuis MD, Brogan DR, Coates RJ, Daling JR, Gammon MD Malone KE, Schoenberg JB, Brinton LA: Hormonal content and potency of oral contraceptives and breast cancer risk among young women. $\mathrm{Br} J$ Cancer 2003, 88:50-57.

29. Brinton LA, Gammon MD, Malone KE, Schoenberg JB, Dalin JR, Coates RJ: Modification of oral contraceptive relationships on breast cancer risk by selected factors among younger women. Contraception 1997, 55:197-203.

30. Collaborative Group on Hormonal Factors in Breast Cancer: Breast cancer and hormonal contraceptives: collaborative reanalysis of individual data on $\mathbf{5 3 , 2 9 7}$ women with breast cancer and 100,239 women without breast cancer from 54 epidemiological studies. Lancet 1996, 347:1713-1727.

31. Moorman PG, Millikan RC, Newman B: Oral contraceptives and breast cancer among African-American women and white women. J Natl Med Assoc 2001, 93:329-334

32. Kumle M, Weiderpass E, Braaten T, Persson I, Adami H-O, Lund E: Use of oral contraceptives and breast cancer risk: the Norwegian-Swedish Women's Lifestyle and Health Cohort Study. Cancer Epidemiol Biomarkers Prev 2002, 11:1375-1381.

33. Dumeaux V, Alsaker E, Lund E: Breast cancer and specific types of oral contraceptives: a large Norwegian cohort study. Int $J$ Cancer 2003, 105:844-850.

34. Wingo PA, Lee NC, Ory HW, Beral V, Peterson HB, Rhodes P: Age-specific differences in the relationship between oral contraceptive use and breast cancer. Obstet Gynecol 1991, 78:161-170

35. Ursin G, Ross RK, Sullivan-Halley J, Hanisch R, Henderson B Bernstein L: Use of oral contraceptives and risk of breast cancer in young women. Breast Cancer Res Treat 1998, 50:175-184

36. Grabrick DM, Hartmann LC, Cerhan JR, Vierkant RA, Therneau TM, Vachon CM, Olson JE, Couch FJ, Anderson KE, Pankratz VS, et al:: Risk of breast cancer with oral contraceptive use in women with a family history of breast cancer. JAMA 2000, 284:1791-1798.

37. Narod SA, Dube MP, Klijn J, Lubinski J, Lynch HT, Ghadirian P, Provencher D, Heimdal K, Moller P, Robson M, et al.: Oral contraceptives and the risk of breast cancer in BRCA1 and BRCA2 mutation carriers. J Natl Cancer Inst 2002, 94:1773-1779.

38. Milne RL, Knight JA, John EM, Dite GS, Balnuena R, Ziogas A, Andrulis IL, West DW, Li FP, Southey MC, for the Breast Cancer Family Registry, et al:: Oral contraceptive use and risk of earlyonset breast cancer in carriers and noncarriers of BRC1 and BRCA2 mutations. Cancer Epidemiol Biomarkers Prev 2005 14:350-356.

39. Jernstrom H, Loman N, Johannsson OT, Borg A, Olsson H: Impact of teenage oral contraceptive use in a population-based series 
of early-onset breast cancer cases who have undergone BRCA mutation testing. Eur J Cancer 2005, 41:2312-2320.

40. Fowke JH, Shu X-O, Dai Q, Jin F, Cai Q, Gao Y-T, Zheng W: Oral contraceptive use and breast cancer risk: modification by $\mathrm{NAD}(\mathrm{P}) \mathrm{H}$ : quinone oxoreductase (NQO1) genetic polymorphisms. Cancer Epidemiol Biomarkers Prev 2004, 13:1308-1315

41. Newcomer LM, Newcomb PA, Trentham-Dietz A, Longnecker MP, Greenberg ER: Oral contraceptive use and risk of breast cancer by histologic type. Int J Cancer 2003, 106:961-964.

42. Li Cl, Malone KE, Porter PL, Weiss NS, Tang MT, Daling JR: Reproductive and anthropometric factors in relation to the risk of lobular and ductal breast carcinoma among women 65-79 years of age. Int J Cancer 2003, 107:647-651.

43. Li Cl, Malone KE, Porter PL, Weiss NS, Tang M-TC, Cushing-Haugen KL, Daling JR: Relationship between long durations and different regimens of hormone therapy and risk of breast cancer. JAMA 2003, 289:3254-3263.

44. Chen C-L, Weiss NS, Newcomb P, Barlow W, White E: Hormone replacement therapy in relation to breast cancer. JAMA 2002, 287:734-741.

45. Isaksson E, von Schoultz E, Odlind V, Soderqvist G, Csemiczky G, Carlstrom K, Skoog L, von Schoultz B: Effects of oral contraceptives on breast epithelial proliferation. Breast Cancer Res Treat 2001, 65:163-169.

46. Olsson $\mathrm{H}$, Jernstrom $\mathrm{H}$, Alm $\mathrm{P}$, Kreipe $\mathrm{H}$, Ingvar $\mathrm{C}$, Jonsson $\mathrm{PE}$, Ryden S: Proliferation of the breast epithelium in relation to menstrual cycle phase, hormonal use and reproductive factors. Breast Cancer Res Treat 1996, 40:187-196.

47. Anderson E, Clarke RB, Howell A: Estrogen responsiveness and control of normal human breast proliferation. I Mammary Gland Biol Neoplasia 1998, 3:23-35.

48. Williams G, Anderson E, Howell A, Watson R, Coyne J, Roberts $S A$, Potten CS: Oral contraceptive (OCP) use increases proliferation and decreases oestrogen content of epithelial cells in the normal human breast. Int J Cancer 1991, 48:206-210.

49. Laidlaw IJ, Clarke RB, Howell A, Owen AW, Potten CS, Anderson $E$ : The proliferation of normal human breast tissue implanted into athymic nude mice is stimulated by estrogen but not progesterone. Endocrinology 1995, 136:164-171.

50. Bramley M, Clarke RB, Howell A, Evans DGR, Armer T, Baildam $A D$, Anderson $E$ : Effects of oestrogens and anti-oestrogens on normal breast tissue from women bearing BRCA1 and BRCA2 mutations. Br J Cancer 2006, 94:1021-1028.

51. Dupont W, Page D: Risk factors for breast cancer in women with proliferative breast disease. N Engl J Med 1985, 312:146-151

52. O'Connell P, Pekkel V, Fuqua SAW, Osborne CK, Clark GM, Allred DC: Analysis of loss of heterozygosity in 399 premalignant breast lesions at $\mathbf{1 5}$ genetic loci. J Nat/ Cancer Inst 1998, 90:697-703.

53. Lakhani SR, Slack DN, Hamoudi RA, Collins N, Stratton MR, Sloane JP: Detection of allelic imbalance indicates that a proportion of mammary hyperplasia of usual type are clonal, neoplastic proliferations. Lab Invest 1996, 74:129-135.

54. Chlebowski and The Women's Health Initiative Steering Committee: Effects of conjugated equine estrogen in postmenopausal women with hysterectomy. The Women's Health Initiative Randomized Controlled Trial. JAMA 2004, 291:1701-1712.

55. Hofseth LJ, Raafat AM, Osuch JR, Pathak DR, Slomski CA, Haslam SZ: Hormone replacement therapy with estrogen or estrogen plus medroxyprogesterone acetate is associated with increased epithelial proliferation in the normal postmenopausal breast. J Clin Endocrinol Metab 1999, 84:4559-4565.

56. Moorman PG, Kuwabara H, Millikan RC, Newman B: Menopausal hormones and breast cancer in a biracial population. $A m \mathrm{~J}$ Public Health 2000, 90:966-971.

57. Newman B, Mu H, Butler LM, Millikan RC, Moorman PG, King MC: Frequency of breast cancer attributable to BRCA1 in a population-based series of American women. JAMA 1998, 279:915-921.

58. Silvera SA, Miller AB, Rohan TE: Oral contraceptive use and risk of breast cancer among women with a family history of breast cancer: a prospective cohort study. Cancer Causes Control 2005, 16:1059-1063. 